\title{
Use of radial artery grafts in extracranial-intracranial revascularization procedures
}

\author{
Hasan Kocaeli, M.D., ${ }^{1}$ Norberto Andaluz, M.D., ${ }^{2}$ Ondrej ChoutKa, M.D., ${ }^{1}$ \\ and Mario ZuCCARELlo, M.D. ${ }^{1,3}$ \\ ${ }^{1}$ Department of Neurosurgery, University of Cincinnati College of Medicine; ${ }^{3}$ The Neuroscience \\ Institute and Mayfield Clinic, Cincinnati, Ohio; and ${ }^{2}$ Department of Neurosurgery, \\ University of South Florida, Tampa, Florida
}

\begin{abstract}
$\checkmark$ Cerebral revascularization procedures have been used in the clinical management of actual or threatened cerebral ischemic states and unclippable cerebral aneurysms. An alternative to a low-flow bypass graft (for example, with the superficial temporal artery) is the use of high-flow grafts created using the saphenous vein (SV) or radial artery (RA). These high-flow grafts are particularly useful when otherwise adequate collateral flow is insufficient to enable sacrifice of the parent vessel without the risk of cerebral ischemia. In their clinical series of 13 patients who underwent highflow bypass with an RA graft, the authors describe 8 women and 5 men whose ages ranged from 44 to 69 years (mean $57.84 \pm 9.05$ years). Indications for RA graft bypass were unclippable aneurysms in 10 patients and occlusive cerebrovascular disease in 3 patients. The authors review the properties of the 2 most common conduits, the SV and RA grafts. They present the technique of high-flow extracranial-intracranial bypass produced using RA grafts in the management of occlusive atherosclerotic disease and complex intracranial aneurysms that are not otherwise amenable to either clip ligation or coil occlusion. (DOI: 10.3171/FOC/2008/24/2/E5)
\end{abstract}

KEY WORDS • cerebral revascularization • high-flow bypass • radial artery graft

A TTEMPTS to restore circulation to the ischemic brain date back to 1942 , when Kredel ${ }^{12}$ reported the use of bilateral temporalis muscle grafts to the brain surface in 3 patients who had suffered a stroke. In the ensuing years, numerous techniques were developed in an effort to establish more effective ways to deliver oxygenated blood to those areas of the brain at risk of ischemia. ${ }^{29}$ Historical milestones in cerebral revascularization are summarized in Table 1.

The use of an RA graft for revascularization procedures was introduced by Carpentier et $\mathrm{al}^{4}{ }^{4}$ for the treatment of coronary artery disease. Potential advantages of RA as a graft conduit include ease of harvesting; low propensity for wound infection; a larger diameter than other arterial grafts; and a thick, muscular wall that facilitates the construction of an anastomosis. Nevertheless, the early experience with the RA was somewhat disappointing, leading to abandon-

Abbreviations used in this paper: $\mathrm{BA}=$ basilar artery; $\mathrm{BTO}=$ balloon test occlusion; $\mathrm{CBF}=$ cerebral blood flow; $\mathrm{CCA}=$ common carotid artery; $\mathrm{CT}=$ computed tomography; $\mathrm{ECA}=$ external carotid artery; EC-IC = extracranial-intracranial; ICA = internal carotid artery; $\mathrm{MCA}=$ middle cerebral artery; $\mathrm{MR}=$ magnetic resonance; $\mathrm{PCA}=$ posterior cerebral artery; PICA = posterior inferior cerebellar artery; RA = radial artery; STA = superficial temporal artery; SV = saphenous vein; $\mathrm{VA}=$ vertebral artery. ment of its use for a long time because of its tendency to develop spasm and functional occlusion. The advent of drug therapy to prevent graft spasms resulted in a renewed interest in the RA as an arterial conduit for grafting. ${ }^{6}$ This technique was first reported by Ausman et al. ${ }^{3}$ in 1978 for the revascularization of a PICA. In 2001, Sekhar et al. ${ }^{22}$ reported on a large series of patients in whom cerebral revascularization was performed using RA grafts.

The indications for EC-IC bypass surgery performed using high-flow grafts typically include complex or giant intracranial or cavernous segment aneurysms of the ICA that are unsuitable for either clipping or endovascular treatment, and complex skull base tumors that incorporate large intracranial vessels (either before planned large-vessel sacrifice, or after failed surgical and/or adjunctive treatment in patients who present with tumor regrowth and large-vessel compromise posing the risk of ischemia). The role of revascularization for atherosclerotic cerebrovascular occlusive disease has been questioned by investigators in an international bypass trial in which they failed to confirm the effectiveness of STA-MCA bypass against best medical treatment. ${ }^{7}$ In the last decade, however, a resurgence of interest in the potential use of EC-IC bypass operations has occurred, governed by the evidence that some patients with occlusion of the ICA have a poor collateral circulation and a high risk for recurrent ischemic events. ${ }^{8,20}$ A new clinical 
TABLE 1

Historical studies of cerebral revascularization*

\begin{tabular}{ll}
\hline \hline \multicolumn{1}{c}{ Authors \& Year } & \multicolumn{1}{c}{ Procedure } \\
\hline Kredel, 1942 & $\begin{array}{c}\text { temporalis muscle grafts laid on brain surface } \\
\text { for revascularization in 3 stroke patients } \\
\text { encephalomyosynangiosis }\end{array}$ \\
$\begin{array}{l}\text { Henschen, 1950 } \\
\text { Welch, 1956 }\end{array}$ & $\begin{array}{l}\text { MCA embolectomy } \\
\text { microsurgical technique, 2-mm-diameter } \\
\text { vacobsel anastomosis }\end{array}$ \\
Woringer \& Kuarez, 1960lin, 1963 & $\begin{array}{l}\text { EC-IC bypass w/ SV graft } \\
\text { PTA-ACA shunt }\end{array}$ \\
Yool \& Potts, 1965 & STA-MCA bypass \\
Lougheed et al., 1971 & CCA-ICA bypass w/ SV graft \\
Sundt et al., 1982 & ECA-PCA bypass w/ SV graft \\
\hline
\end{tabular}

$* \mathrm{ACA}=$ anterior cerebral artery.

trial-The Carotid Occlusion Surgery Study, which is being funded by the National Institutes of Health—will test the hypothesis that bypass procedures may be of benefit in patients with carotid artery occlusion with demonstrated hemodynamic-dependent mechanisms of stroke. ${ }^{9}$

In this retrospective review, we will present the technique of high-flow EC-IC bypass in which an RA graft was used in our clinical series of 13 patients. We describe our stepwise protocol and then compare the properties, indications, and outcomes of the 2 most commonly used graft conduits, the SV and the RA.

\section{Patient Series}

Between December 2003 and September 2007, 81 pa- tients underwent cerebral revascularization procedures performed by the senior author (M.Z.), including 68 patients with STA-MCA bypasses and 13 with high-flow bypasses performed with an RA graft (Table 2). The 13 patients consisted of 8 women and 5 men whose ages ranged from 44 to 69 years (mean $57.84 \pm 9.05$ years). Two patients presented with a ruptured aneurysm. Nine patients underwent an ECA-MCA bypass, 2 underwent ECA-PCA bypass, 1 underwent ECA-VA/PICA bypass, and 1 underwent a subclavian artery-MCA bypass because of complete occlusion of the CCA. The indications for RA graft bypass were unclippable aneurysms in 10 patients and occlusive cerebrovascular disease in 3. Among the 10 patients with unclippable aneurysms, location included the cavernous and supraclinoid ICA in 8 patients, anterior communicating artery in 1, and giant BA in 1. Among the 3 patients with occlusive vascular disease, occlusion was located in the ICA in 1 and in the VA bilaterally in 2 patients.

Clinical and radiological follow-up duration ranged from 2 months to 3 years; all patients had a patent bypass graft at the last follow-up appointment. Two patients suffered a minor stroke with nearly complete recovery at follow-up and 1 suffered a stroke in the distribution of the inferior branch of the PCA.

\section{Illustrative Cases}

A summary of the clinical cases appears in Table 2.

Case 6

This 53-year-old woman presented to our service with a

TABLE 2

Summary of 13 patients who underwent revascularization with RA graft between 2003 and 2007*

\begin{tabular}{|c|c|c|c|c|c|}
\hline $\begin{array}{l}\text { Case } \\
\text { No. }\end{array}$ & $\begin{array}{l}\text { Age (yrs), } \\
\text { Sex }\end{array}$ & Indications & Procedure & $\begin{array}{l}\text { 30-Day M \& M } \\
\text { Findings }\end{array}$ & FU, Graft Patency, \& Outcome \\
\hline 1 & $69, \mathrm{~F}$ & $\begin{array}{l}\text { 1t CS syndrome, 1t ICA cavernous } \\
\text { segment giant aneurysm }\end{array}$ & 1t ECA-MCA bypass, trapping of 1t ICA & improved & 3 yrs, patent, normal exam results \\
\hline 2 & $69, \mathrm{~F}$ & SAH, rt giant OphA aneurysm & rt ECA-MCA bypass, trapping of ICA & $\begin{array}{l}\text { lt hemiparesis, } \\
\mathrm{A}_{1} \text { small stroke }\end{array}$ & 2 yrs, patent, normal exam results \\
\hline 3 & $67, \mathrm{~F}$ & rt ICA occlusion at origin & rt subclavian-MCA bypass & $\begin{array}{l}\text { lt upper extremity } \\
\text { Grade } 4 / 5\end{array}$ & 2 yrs, patent, mild $1 t$ hemiparesis \\
\hline 4 & $62, \mathrm{M}$ & rt giant ICA aneurysm & rt ECA-MCA bypass, trapping of ICA & $\begin{array}{l}\text { rt minor parietal } \\
\text { stroke, lt mild } \\
\text { hemiparesis }\end{array}$ & 3 yrs, patent, mild lt hemiparesis \\
\hline 5 & $65, \mathrm{~F}$ & $\begin{array}{l}\text { rt CS syndrome, rt giant } \\
\text { cavernous segment aneurysm }\end{array}$ & $\begin{array}{l}\text { rt ECA-MCA bypass, coil occlusion } \\
\text { of ICA }\end{array}$ & stable & $\begin{array}{l}1 \text { yr, patent, improved CS } \\
\text { syndrome }\end{array}$ \\
\hline 6 & $53, \mathrm{~F}$ & $\begin{array}{l}\text { rt CS syndrome, rt giant cavernous } \\
\text { segment aneurysm }\end{array}$ & $\begin{array}{l}\text { rt ECA-MCA bypass, coil occlusion } \\
\text { of ICA }\end{array}$ & stable & 3 yrs, patent, normal exam results \\
\hline 7 & $64, \mathrm{M}$ & $\begin{array}{l}\text { PCA \& brainstem stroke, bilat } \\
\text { VA occlusion }\end{array}$ & rt ECA-PCA bypass & stable & $\begin{array}{l}6 \text { mos, patent, neurologically } \\
\text { unchanged }\end{array}$ \\
\hline 8 & $58, \mathrm{~F}$ & $\begin{array}{l}\text { rt CS syndrome, rt giant cavernous } \\
\text { segment aneurysm }\end{array}$ & rt ECA-MCA bypass, trapping of ICA & stable & 3 yrs, patent, normal exam results \\
\hline 9 & $45, \mathrm{M}$ & $\begin{array}{l}\text { occluded rt ICA, fusiform ACoA } \\
\text { aneurysm }\end{array}$ & rt ECA-MCA bypass & stable & $1 \mathrm{yr}$, patent, normal exam results \\
\hline 10 & $57, \mathrm{~F}$ & VBA ischemia, bilat VA stenosis & rt ECA-VA/PICA bypass & stable & 2 yrs, patent, normal exam results \\
\hline 11 & $46, \mathrm{M}$ & SAH, lt fusiform ICA aneurysm & 1t ECA-MCA bypass & stable & $1 \mathrm{yr}$, patent, normal exam results \\
\hline 12 & $44, \mathrm{~F}$ & lt fusiform ICA aneurysm & lt ECA-MCA bypass & stable & $1 \mathrm{yr}$, patent, normal exam results \\
\hline 13 & $53, \mathrm{M}$ & $\begin{array}{l}\text { dizziness, lt CN VI palsy, giant } \\
\text { BA aneurysm }\end{array}$ & rt ECA-PCA bypass & $\begin{array}{l}\text { rt PCA branch } \\
\text { stroke }\end{array}$ & 2 mos, patent, lt hemiparesis \\
\hline
\end{tabular}

* $\mathrm{ACoA}=$ anterior communicating artery; $\mathrm{CN}=$ cranial nerve; $\mathrm{CS}=$ cavernous sinus; $\mathrm{FU}=$ follow-up; $\mathrm{M} \& \mathrm{M}=$ morbidity and mortality; OphA = ophthalmic artery; $\mathrm{SAH}=$ subarachnoid hemorrhage; VBA = vertebrobasilar artery. 

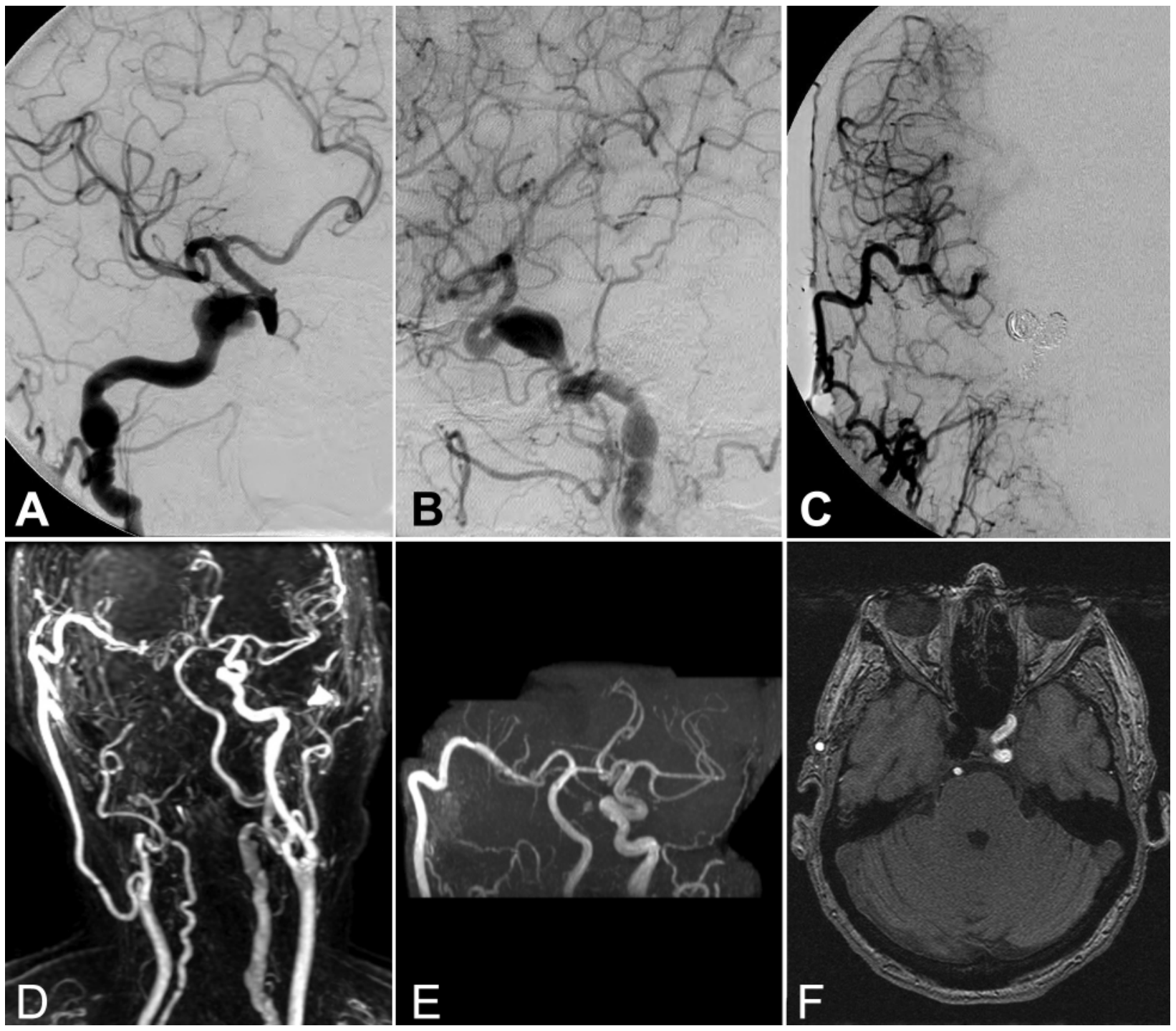

FIG. 1. Case 6. Cavernous ICA aneurysm treated with an ECA-MCA bypass for which an RA graft was used. Preoperative anteroposterior (A) and lateral (B) oblique view angiograms demonstrating a partially thrombosed aneurysm of the cavernous segment of the right ICA. Postoperative conventional (C) and MR angiograms (D and E) demonstrating a patent ECA-MCA $\left(\mathrm{M}_{2}\right.$ segment) bypass, and coil occlusion of the right ICA. An MR angiogram (F) obtained at the 3-year follow-up evaluation demonstrates that the bypass is patent and the aneurysm is completely thrombosed. (Reprinted with permission from the Mayfield Clinic; originally published at http://www.mayfieldclinic.com/ DNS/ClinTrial_COSS.htm.)

progressive onset of cavernous sinus syndrome on the right side. She had partial third cranial nerve palsy and complete sixth cranial nerve palsy. The MR imaging study and cerebral angiogram showed the presence of a giant unclippable cavernous carotid segment ICA aneurysm (Fig. 1). After failure of the BTO, she underwent ECA-MCA $\left(\mathrm{M}_{2}\right.$ segment) bypass with the RA graft. At the 3-year follow-up examination, she was neurologically intact with a patent bypass graft.

\section{Case 10}

This 57-year-old woman presented to our service with a 6-month history of intractable vertigo and episodes of brief loss of consciousness. Cerebral angiograms revealed the presence of bilateral occlusion of the VAs and insufficient collateral flow from the posterior communicating arteries. Although the patient was treated with maximal medical therapy, which consisted of antiplatelet and anticoagulation therapy, she continued to have symptoms. After a decision was made to proceed with cerebral revascularization, she underwent an ECA-VA/PICA bypass with the RA graft, and her symptoms resolved (Fig. 2).

\section{Discussion}

Our clinical series follows 13 patients who underwent a high-flow bypass with an RA graft for treatment of either unclippable aneurysms or occlusive cerebrovascular disease. At follow-up evaluation, all patients had grafts that 

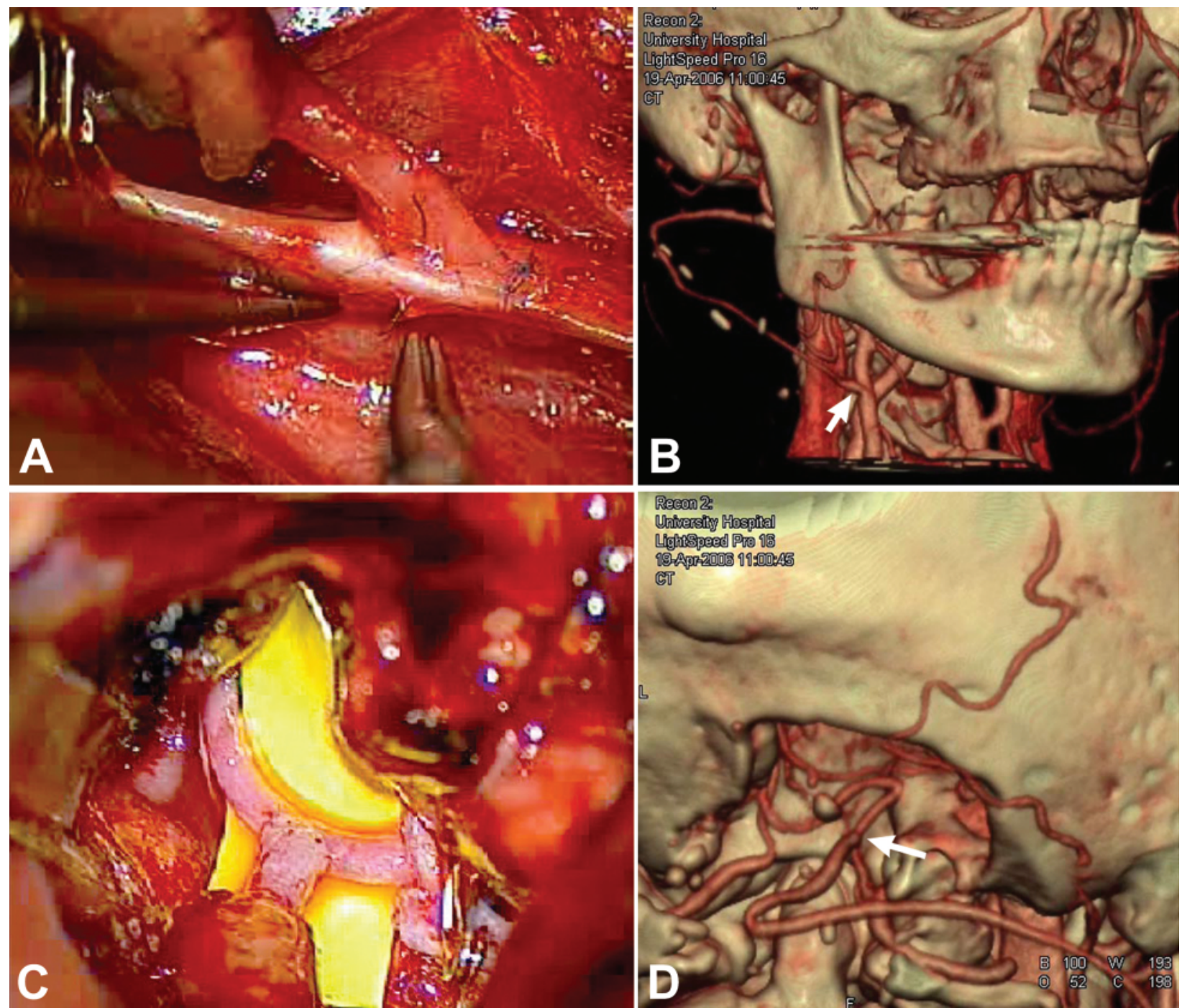

FIG. 2. Case 10. Bilateral VA occlusions treated with an ECA-VA/PICA bypass in which an RA graft was used. Intraoperative photograph (A) and postoperative CT angiogram (B) of the proximal end-to-side anastomosis of the RA graft to the ECA (arrow). Intraoperative photograph (C) and postoperative CT angiogram (D) of the distal end-to-side anastomosis of the RA graft to VA/PICA (arrow). At the 1-year follow-up evaluation, the bypass is patent at both cervical and intracranial sides. (Reprinted with permission from the Mayfield Clinic; originally published at http: //www. mayfieldclinic.com/DNS/ClinTrial_COSS.htm.)

remained patent. The stepwise procedure combines our clinical experience with contemporary reports in the literature on this topic. We review the need for collaboration with vascular specialists in graft selection (RA as opposed to SV), placement, and monitoring; diagnostic assessment of cerebral circulation and follow-up testing; operative steps; and postoperative care of the patient.

\section{Preoperative Evaluations}

Graft Evaluation. Of great importance during preoperative preparation is the evaluation of the vascular conduit by a cardiovascular surgery team. Patients undergo evaluation of both forearms and legs in the vascular laboratory, specifically to measure the length of both RAs and to conduct an Allen test as an indicator of the collateral flow. Because the length of the graft is the critical aspect of RA harvesting and can be the major limiting factor for surgery, we always ask for mapping of the SVs preoperatively.

Imaging Evaluation. Planned arterial occlusion includes proximal parent artery occlusion, distal occlusion, trapping, and reversal of blood flow through an aneurysm. The safety of planned arterial occlusion depends on a careful assessment of the patient's cerebral circulation. Although cerebral angiography is an important evaluation in patients with occlusive cerebrovascular disease, 3D CT angiography is the test of choice for cerebral aneurysms (Fig. 3). Collateral circulation on a diagnostic angiogram and tolerance to the planned occlusion with a BTO are assessed. Angiography examines collateral circulation in the leptomeningeal vessels and circle of Willis, but it cannot predict whether a patient will tolerate an arterial occlusion. Therefore, the BTO administered with a hypotensive challenge and CBF studies are used for predicting tolerance. 

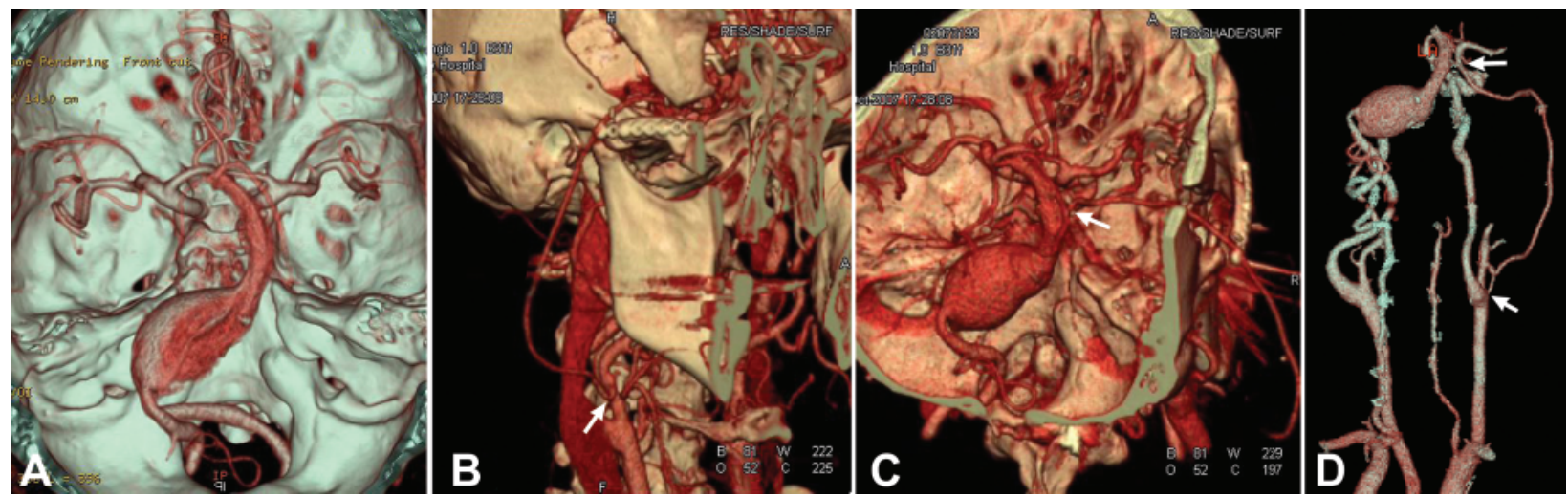

FIG. 3. The 3D CT angiography study is the test of choice for cerebral aneurysms. Preoperative image (A) reveals a giant BA aneurysm. Postoperative images of the proximal (B) and distal (C) anastomoses (arrows) of a patent ECAPCA bypass performed before occlusion of the right VA (D). (Reprinted with permission from the Mayfield Clinic; originally published at http: //www.mayfieldclinic.com/DNS/A_Cerebro.htm.)

\section{The BTO Protocol}

At the University of Cincinnati, a temporary BTO protocol has been developed (Fig. 4). ${ }^{2}$ The protocol consists of test arterial occlusion with a balloon-tip catheter and neurological examination for 30 minutes, during which distal arterial back pressures are measured as an indirect indicator of collateral circulation. When conventional BTO is successful, patients subsequently undergo a hypotensive challenge by reduction of the mean arterial pressure by 25-30\% and continuous neurological testing. Technetiumhexamethylpropyleneamine oxime single-photon emission $\mathrm{CT}$ imaging is performed to assess asymmetry in CBF during temporary balloon occlusion.

Patients in whom the conventional BTO fails are candidates for revascularization if direct treatment of the aneurysms would result in deliberate arterial sacrifice. With these patients, a high-flow EC-IC bypass is typically needed to replace the blood flow that will be lost with intervention. Patients who tolerate conventional BTO but have either low back-pressures, neurological deterioration with hypotensive challenge, or perfusion defects on the $\mathrm{CBF}$ study are also candidates for bypass procedures if direct treatment of the aneurysms would result in arterial sacrifice. These patients may need only a low-flow bypass, such as an STA as the donor artery or an in situ donor artery. Nevertheless, BTO may not reliably predict the capacity of the collateral flow after occlusion of the ICA. Negative results on the BTO have been associated with delayed ischemic events in up to $20 \%$ of patients. ${ }^{23}$

\section{Anesthesia and Monitoring}

Aspirin therapy ( $325 \mathrm{mg}$ daily) is used for 7 days before surgery. In the presence of hypercholesterolemia, administration of a statin is advisable because of its favorable longterm effect on the graft patency. ${ }^{13}$ Prophylactic antibiotics and monitoring of the central venous and arterial lines are routine. Intraoperative neurophysiological monitoring is performed, which includes continuous electroencephalography, somatosensory evoked potentials, and brainstem evoked potentials. Monitoring of the cranial nerves for those that may be at risk during exposure is advisable. A femoral artery sheath is inserted for intraoperative angiography.

The anastomosis site is selected on the basis of several factors: the intended revascularization territory, size of donor and recipient vessels, length of the graft, location of the lesion, and extension of the lesion within the sylvian fissure. The site of graft extraction is marked before the operation to avoid placing the RA line in that arm. Brain relaxation is achieved with the use of lumbar or ventricular drainage, in addition to the administration of intraoperative mannitol. Appropriately balanced neuroanesthetic techniques are used. In patients with ruptured aneurysms, normal blood pressure and volume are maintained during burst suppression. In cases of unruptured aneurysms, the blood pressure is elevated $20 \%$ above the patient's normal value. Intraoperative micro-Doppler monitoring (Mizuho America, Inc.) is used as a fast qualitative means to assess flow within the graft. Intraoperative cerebral angiography is always performed before completion of the surgical procedure.

\section{Choice of Graft}

Arterial Compared With Venous Grafts. In a 2001 study, Shi et al. ${ }^{24}$ reported on the biology of venous compared with arterial grafts in a porcine model of graft interposition into the carotid artery. The authors found significant differences between the biology of venous and arterial grafts. Venous grafts showed higher oxidative stress, accumulation of low-density lipoprotein, and the presence of oxidized epitopes. Furthermore, proatherogenic changes were present in the venous graft early after revascularization, thus creating conditions for the development of graft thrombosis. These findings then provided the biological rationale for the use of arterial conduits (for example, RA graft) for cerebral revascularization.

The RA Graft. The advantages of using an RA graft are its ease of harvesting and its luminal caliber that closely approximates the usual recipient (for example, MCA or PCA). The diameter of the RA is $3.55 \pm 0.45 \mathrm{~mm}$, which is more compatible with the diameter of the $\mathrm{M}_{2}$ or $\mathrm{P}_{1}$ branches. ${ }^{14}$ Another benefit of the RA graft is the absence 


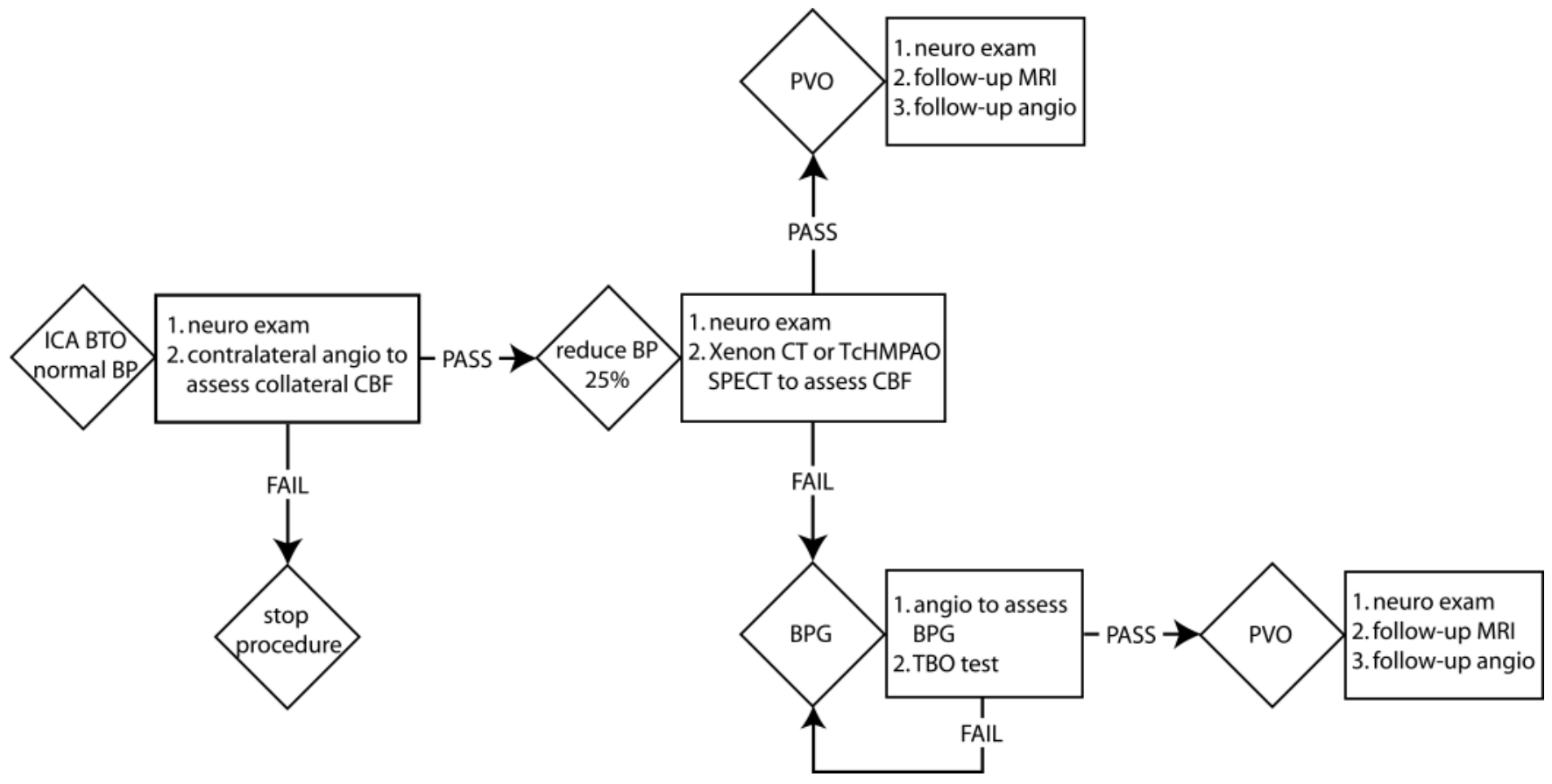

FIG. 4. Algorithm showing the protocol for BTO in the treatment of large and giant unclippable aneurysms. angio = angiography; $\mathrm{BP}=$ blood pressure; $\mathrm{BPG}=$ bypass graft; neuro = neurological; $\mathrm{PVO}=$ permanent vessel occlusion; SPECT = single-photon emission computed tomography; TBO = temporary balloon occlusion; TcHMPAO = technetium-hexamethylpropyleneamine oxime. (Reprinted with permission from the Mayfield Clinic; originally published in Link MJ, Tomsick TA, Tew JM Jr: Honored guest presentation: therapeutic carotid occlusion. Clin Neurosurg 46: $326-338,2000$.

of valves and varices that can predispose the graft to occlusion with thrombus during manipulation..$^{19}$ As the RA provides a flow rate between 50 and $150 \mathrm{ml} /$ minute initially, transcranial Doppler flowmetry shows that this flow can increase significantly within days after surgery. ${ }^{21}$

The most serious disadvantage of an RA graft is the presence of arterial spasms; however, these can be partially resolved with the aid of a calcium channel antagonist and endovascular angioplasty. Sekhar and colleagues ${ }^{21,22}$ described an alternative in the pressure distension technique that minimizes spasm during the extraction of the graft; however, its potential disadvantage includes intravascular endothelial injury caused by denervation or disruption of the vasa vasorum. Another potential disadvantage of RA graft use is intimal hyperplasia. ${ }^{5}$

The SV Graft. These grafts are preferred in some circumstances when the RA is either an unsuitable vessel or when it has become occluded during the perioperative period. The SV has a much thicker wall than the intracranial vessels, and because of the high flow through it, is more prone to kinking at the distal anastomotic site. Anastomosis may be technically more difficult to perform with the SV. Flow in this SV graft has ranged from 100 to $200 \mathrm{ml} /$ minute. In children younger than 12 years of age, the SV graft is the only alternative because the RA graft is too small in diameter. A flow mismatch can occur when the SV graft with its high flow is anastomosed into vessels $<2 \mathrm{~mm}$ in diameter; this mismatch can cause kinking because of excessive and turbulent flow. ${ }^{16}$

\section{Graft Patency}

The long-term patency rate for RA grafts has not been reported in the neurosurgery literature. However, 10-year patency rates exceeding $90 \%$ have been reported in patients who underwent coronary revascularization procedures. ${ }^{18}$ In a prospective study, Desai et al. ${ }^{6}$ suggested that the rate of graft occlusion at 1 year is lower in RA than in SV grafts.

\section{Surgical Technique}

The following section describes our technique of highflow EC-IC bypass with an RA graft that we used in our 13 patients.

\section{Patient Positioning}

Patient positioning is tailored according to the side of vessel occlusion or the location of the aneurysm, the donor and recipient vessel, and the site of graft harvesting. For anterior circulation aneurysms, the patient is placed supine and the head is turned away from the lesion site as necessary. For posterior circulation lesions, a park bench position is usually used and care is taken not to turn the neck excessively, which can compress the VA at the C1-2 level. A femoral artery sheath is inserted before placing the patient in the lateral position. The RA is harvested from the recumbent forearm when the patient is in the lateral position.

\section{Cervical Exposure}

After review of the cerebral angiogram or CT angiogra- 
phy study to identify the level of the CCA bifurcation, a 5$\mathrm{cm}$-long incision is made along the anterior edge of the sternocleidomastoid muscle. The muscle is dissected from the skin and retracted. With continued deep dissection, the digastric muscle and the hypoglossal nerve, which cross the ICA, are identified. The CCA, ICA, and ECA are then each exposed for a length of at least $2 \mathrm{~cm}$, separating them from the internal jugular vein. The next step is to isolate the CCA, ICA, and ECA with vessel loops. We always plan to use the ECA as the site of proximal anastomosis. This vessel is exposed up to $4-5 \mathrm{~cm}$ from the CCA bifurcation to permit sufficient space for temporary clip application and anastomosis.

\section{Craniotomy Parameters}

The type and size of the craniotomy are dictated by the indication of the revascularization. For ECA-MCA bypass, a standard frontotemporal pterional craniotomy is typically used. Occasionally, a cranial base approach, such as a frontotemporal-orbitozygomatic approach or its variants, is used for giant aneurysms. Before the dural opening, epidural bleeding must be carefully controlled, because heparin is administered intravenously during the procedure (heparin at a dose of $1500 \mathrm{U}$ is administered intravenously as a single bolus and not as a continuous infusion). Thinning of the zygomatic arch posteriorly with a high-speed drill allows the RA to lie without kinking at the zygomatic area.

\section{Harvesting of the RA}

The neurosurgical and cardiovascular surgery teams collaborate in the planning and harvesting of the RA graft. The length of the graft is the critical aspect of RA harvesting because it can be a limiting factor leading to abandonment of the procedure. Specifically, the teams discuss the expected length needed and plan for a scenario in which additional length may be needed so that further proximal or distal dissection in the forearm can be made before clamping of the vessel. Harvest of the RA graft is done at the same time as the splitting of the sylvian fissure. The superficial wall of the RA is identified with a marking pen before removal of the graft to avoid its rotation during the tunneling phase of the operation.

After harvest, the graft is placed in a heparinized saline solution and is cannulated with a blunt needle. The RA graft is then fully expanded and filled with a solution containing heparin and calcium channel blockers. This step confirms absence of leakage, prevents vasospasm, and avoids distortion of the graft. Then, the adventitia is removed from both ends of the graft $(\sim 1-1.5 \mathrm{~cm}$ each).

\section{Tunneling the Graft}

The tunnel is started below the zygoma, immediately anterior to the root of the zygomatic process of the temporal bone. A hemostatic clamp with a blunt end or a pair of curved scissors is used to penetrate the insertion of the temporalis muscle in front of the condyle of the jaw. The clamp is then routed laterally, following the ascending branch of the mandible until reaching its angle. Next, the clamp perforates the insertion of the masseter muscle and parotid fascia, and ends in the anterior-superior portion of the cervical incision. After a pediatric chest tube is introduced in the tunnel in the cephalad direction, the tube remains in place to form a rigid tunnel for the passage of the graft. The marked surface of the graft is kept mostly superficial.

\section{Intracranial Anastomosis}

We prefer to perform the intracranial anastomosis first. Thus, the surgeon can take advantage of the slack in the graft, manipulating it freely while the back and front walls are sutured. Although we strive to keep the time of anastomosis $<30$ minutes, an ECA-PCA bypass can take as much as 50-55 minutes. A color background is placed behind the recipient vessel. After application of temporary clips, an arteriotomy made using a diamond knife (Aesculap, Inc.) is then extended using microscissors without excision of any of the arterial wall. The length is comparable to the donor vessel lumen, which rarely exceeds 3-3.5 $\mathrm{mm}$. The lumen of the donor vessel is expanded if necessary by "fish-mouthing" the opening. Preferring an interrupted suture technique, we use a 9-0 nylon for $\mathrm{M}_{2}-\mathrm{M}_{3}$ and $\mathrm{P}_{2}-\mathrm{P}_{3}$ vessels, and 8-0 nylon for the supraclinoid ICA. Before completion of the anastomosis, the graft is flushed with heparinized solution, and a temporary clip is placed on the graft just distal to the anastomosis. On completion of the anastomosis, retrograde flow is confirmed in the graft, rotation and twisting are avoided, and a temporary clip is reapplied.

\section{Extracranial Anastomosis}

Anastomosis is made in an end-to-side manner between the ECA and the RA graft. After circumferential removal of the adventitia, the end is cut in fish-mouth form to increase the area of anastomosis. All the branches of the ECA are temporarily ligated with clips, the ECA is crossclamped with 2 long temporary aneurysm clips, and the graft is flushed with heparinized solution to prevent thrombosis. An arteriotomy is made using a diamond knife; this opening is extended using microscissors without excising any of the arterial wall, so that its length will match the lumen of the RA. The anastomosis is then performed with 8-0 prolene sutures applied in an interrupted manner. Before closing the last knot, the flow is reestablished to avoid arterial embolism. The ECA is then clamped and the last knot is closed. Clamps are removed in the following sequence: distal portion of the recipient artery, proximal portion of the recipient artery, distal segment of the ECA, and finally proximal segment of the ECA. Once the flow is reestablished, bleeding points are identified. FloSeal (Baxter International, Inc.) is usually sufficient to control small bleeding points; otherwise, more stitches are applied.

\section{Graft Inspection (Doppler Ultrasonography, Intraoperative Angiography)}

The adequacy of blood flow through the graft after the restoration of circulation to the ECA must be confirmed. Pulsatility of the graft is not enough to confirm its patency. Micro-Doppler ultrasonography (Mizuho America, Inc.) can be used. New flow probe technology (Transonic Systems, Inc.) has been used to estimate the amount of flow within the STA and occipital graft. Amin-Hanjani et al. ${ }^{1}$ suggested the use of the cut flow index as follows: bypass 
function $=$ flow $(\mathrm{ml} /$ minute $) /$ cut flow $(\mathrm{ml} /$ minute $)$. Using a cut flow index of $\geq 0.5$ as a threshold, the authors reported that the bypass patency rate was $92 \%$ in patients with an index $\geq 0.5$ compared with $50 \%$ in patients with an index $<0.5$. They found good correlation between intraoperative bypass flow and postoperative measurements obtained from quantitative phase-contrast MR imaging. Nevertheless, this technology has yet to be used in the evaluation of high-flow bypasses.

We continue to recommend intraoperative angiography as a critical portion of the graft patency assessment, particularly when parent vessel occlusion is planned. Our practice is to occlude the parent vessel during the same surgical procedure rather than to delay this step. The proximal ICA is ligated just above the bifurcation of the CCA and the supraclinoid ICA is clipped proximal to the posterior communicating artery. However, in some patients with giant cavernous segment ICA aneurysms, the ICA is occluded by coil insertion. Because the length of the graft is the critical aspect of RA harvesting and can be the major surgical limiting factor, we always ask for mapping of the SVs preoperatively.

\section{Postoperative Care}

Postoperative monitoring of graft patency usually consists of palpation of the graft and Doppler evaluation. Postoperatively, we check the graft every 4 hours during Day 1, every 8 hours during Day 2, and then twice a day until discharge. The CT angiography study to evaluate the graft is usually performed 24 to 36 hours postoperatively. If there is concern regarding the functioning of the graft, intraarterial angiography is performed again. Autoregulation of CBF may be impaired or absent, particularly in ischemic patients in whom high-flow bypasses are used for revascularization. Therefore, systemic hypertension $(>20 \mathrm{~mm}$ above the baseline) must be avoided for 48 hours after the operation. The cardiovascular surgery team checks the hand vascularization for the first 24 hours. Patients are maintained on subcutaneously administered heparin (5000 $\mathrm{U}$ every 8 hours for 3 days) and aspirin (325 mg once daily). After discharge, the patients continue a daily regimen of $325 \mathrm{mg}$ of aspirin indefinitely. The graft is followed postoperatively by $3 \mathrm{D} C \mathrm{CT}$ angiography or MR angiography at 3 months, 1 year, and every 2 years thereafter. After 5 years, radiographic follow-up is discontinued unless symptoms recur.

\section{Conclusions}

In our case series, the RA proved to be a useful graft for cerebral revascularization. Its long-term patency has been demonstrated in the surgical management of giant aneurysms of the cavernous and paraclinoid ICAs. The RA has a lumen that closely approximates that of the $\mathrm{M}_{2}$ segment, and it has the advantage of being a physiological conduit for arterial blood. Unlike venous grafts, the RA lacks valves and varices, and is technically easier to harvest because of its consistent anatomical location and size. More resistant to kinking than the SV, the RA is preferable to this vein, whose flow mismatch can lead to subsequent flow turbulence and graft thrombosis. The RA grafts can better tolerate intermittent temporary occlusion when compared with vein grafts, whose valves and endothelium can promote thrombosis in low-flow states.

\section{Disclaimer}

None of the authors has any investment or financial relationships with the manufacturer of any of the commercial products mentioned in this report.

\section{References}

1. Amin-Hanjani S, Du X, Mlinarevich N, Meglio G, Zhao M, Charbel FT: The cut flow index: an intraoperative predictor of the success of extracranial-intracranial bypass for occlusive cerebrovascular disease. Neurosurgery 56 (1 Suppl):75-85, 2005

2. Andaluz N, Beretta F, Keller JT, Zuccarello M: Aneurysms of the ophthalmic (C6) segment of the internal carotid artery. Clinical experience, treatment options, and strategies (part 2). Neurosurg 15:91-102, 2006

3. Ausman JI, Nicoloff DM, Chou SN: Posterior fossa revascularization: anastomosis of vertebral artery to PICA with interposed radial artery graft. Surg Neurol 9:281-286, 1978

4. Carpentier A, Guermonprez JL, Deloche A, Frechette C, DuBost $\mathrm{C}$ : The aorta-to-coronary radial artery bypass graft. A technique avoiding pathological changes in grafts. Ann Thorac Surg 16: $111-121,1973$

5. Curtis JJ, Stoney WS, Alford WC Jr, Burrus GR, Thomas CS Jr: Intimal hyperplasia. A cause of radial artery aortocoronary bypass graft failure. Ann Thorac Surg 20:628-635, 1975

6. Desai ND, Cohen EA, Naylor CD, Fremes SE, Radial Artery Patency Study Investigators: A randomized comparison of radialartery and saphenous-vein coronary bypass grafts. N Engl J Med 351:2302-2309, 2004

7. EC/IC Bypass Study Group: Failure of extracranial-intracranial arterial bypass to reduce the risk of ischemic stroke. Results of an international randomized trial. N Engl J Med 313:1191-1200, 1985

8. Grubb RL Jr, Derdeyn CP, Fritsch SM, Carpenter DA, Yundt KD, Videen TO, et al: Importance of hemodynamic factors in the prognosis of symptomatic carotid occlusion. JAMA 280:1055-1060, 1998

9. Grubb RL Jr, Powers WJ, Derdeyn CP, Adams HP Jr, Clarke WR: The Carotid Occlusion Surgery Study. Neurosurg Focus 14(3): E9, 2003

10. Henschen C: [Surgical revascularization of cerebral injury of circulatory origin by means of stratification of pedunculated muscle flaps.] Langenbecks Arch Klin Chir Ver Dtsch Z Chir 264: 392-401, 1950 (Ger)

11. Jacobson JH, Suarez E: Microsurgery in anastomosis of small vessels. Surg Forum 11:243-245, 1960

12. Kredel FE: Collateral cerebral circulation by muscle graft. Technique of operation with report of 3 cases. South Surgeon 10: 235-244, 1942

13. Lazar HL: Role of statin therapy in the coronary bypass patient. Ann Thorac Surg 78:730-740, 2004

14. Liu JK, Kan P, Karwande SV, Couldwell WT: Conduits for cerebrovascular bypass and lessons learned from the cardiovascular experience. Neurosurg Focus 14(3):E3, 2003

15. Lougheed WM, Marshall BM, Hunter M, Michel ER, SandwithSmyth H: Common carotid to intracranial internal carotid bypass venous graft. Technical note. J Neurosurg 34:114-118, 1971

16. Mohit AA, Sekhar LN, Natarajan SK, Britz GW, Ghodke B: High-flow bypass grafts in the management of complex intracranial aneurysms. Neurosurgery 60 (1 Suppl):ONS105-ONS123, 2007

17. Pool JL, Potts DG: Aneurysms and Arteriovenous Anomalies of the Brain: Diagnosis and Treatment. New York: Hoeber Medical Division, 1965, p 49

18. Possati G, Gaudino M, Prati F, Alessandrini F, Trani C, Glieca F, 


\section{Radial artery graft in revascularization}

et al: Long-term results of the radial artery used for myocardial revascularization. Circulation 108:1350-1354, 2003

19. Quiñones-Hinojosa A, Du R, Lawton MT: Revascularization with saphenous vein bypasses for complex intracranial aneurysms. Skull Base 15:119-132, 2005

20. Schmiedek P, Piepgras A, Leinsinger G, Kirsch CM, Einhüpl K: Improvement of cerebrovascular reserve capacity by EC-IC arterial bypass surgery in patients with ICA occlusion and hemodynamic cerebral ischemia. J Neurosurg 81:236-244, 1994

21. Sekhar LN, Bucur SD, Bank WO, Wright DC: Venous and arterial bypass grafts for difficult tumors, aneurysms, and occlusive vascular lesions: evolution of surgical treatment and improved graft results. Neurosurgery 44:1207-1224, 1999

22. Sekhar LN, Duff JM, Kalavakonda C, Olding M: Cerebral revascularization using radial artery grafts for the treatment of complex intracranial aneurysms: techniques and outcomes for 17 patients. Neurosurgery 49:646-659, 2001

23. Sekhar LN, Patel SJ: Permanent occlusion of the internal carotid artery during skull base and vascular surgery: is it really safe? Am J Otol 14:421-422, 1993

24. Shi Y, Patel S, Davenpeck KL, Niculescu R, Rodriguez E, Magno $\mathrm{MG}$, et al: Oxidative stress and lipid retention in vascular grafts: comparison between venous and arterial conduits. Circulation 103:2408-2413, 2001
25. Sundt TM, Piepgras DG, Houser OW, Campbell JK: Interposition saphenous vein grafts for advanced occlusive disease and large aneurysms in the posterior circulation. J Neurosurg 56:205-215, 1982

26. Welch K: Excision of occlusive lesions of the middle cerebral artery. J Neurosurg 13:73-80, 1956

27. Woringer E, Kunlin J: [Anastomosis between the common carotid and the intracranial carotid or the sylvian artery by a graft, using the suspended suture technique.] Neurochirurgie 200:181-188, 1963 (Fr)

28. Yaşargil MG: Anastomosis between the superficial temporal artery and a branch of the middle cerebral artery, in Microsurgery Applied to Neurosurgery. New York: Academic Press, 1969, pp 105-115

29. Yaşargil MG, Krayenbühl HA, Jacobson JH II: Microneurosurgical arterial reconstruction. Surgery 67:221-233, 1970

Manuscript submitted November 15, 2007.

Accepted December 10, 2007.

Address correspondence to: Mario Zuccarello, M.D., c/o Editorial Office, Department of Neurosurgery, University of Cincinnati College of Medicine, ML 0515, 231 Albert Sabin Way, Cincinnati, Ohio 45267-0515. email: editor@ mayfieldclinic.com. 